\title{
Correction: Ankyrin-G regulates forebrain connectivity and network synchronization via interaction with GABARAP
}

\author{
A. D. Nelson ${ }^{1} \cdot$ R. N. Caballero-Florán ${ }^{1} \cdot$ J. C. Rodríguez Díaz ${ }^{2} \cdot$ J. M. Hull ${ }^{1,2} \cdot$ Y. Yuan ${ }^{1} \cdot$ J. Li $\mathbb{D}^{3} \cdot$ K. Chen ${ }^{3} \cdot$ \\ K. K. Walder ${ }^{4}$ L. F. Lopez-Santiago ${ }^{1} \cdot$ V. Bennett ${ }^{4,5} \cdot$ M. G. Mclnnis $\mathbb{D}^{6} \cdot$ L. L. Isom ${ }^{1,2,7,8} \cdot$ C. Wang $\mathbb{D}^{3,9} \cdot$ M. Zhang $\mathbb{D}^{3} \cdot$ \\ K. S. Jones ${ }^{1,2} \cdot$ P. M. Jenkins $\mathbb{1}^{1,6}$
}

Published online: 31 January 2019

(c) Springer Nature Limited 2019

\section{Correction to: Molecular Psychiatry; https://doi.org/10.1038/s41380-018-0308-x; published online 30 November 2018}

In the original version of this article, affiliation 3 was given as: "Division of Life Sciences, State Key Laboratory of Molecular Neuroscience, Hong Kong, University of Science and Technology, Clear Water Bay, Kowloon, Hong Kong, China". This has now been corrected to: "Division of
Life Sciences, State Key Laboratory of Molecular Neuroscience, Hong Kong University of Science and Technology, Clear Water Bay, Kowloon, Hong Kong, China".

Additionally in the 'Data availability' section an incorrect accession code was given. The accession code has now been changed from 'PDB A9X (AnkG:GABARAPL)' to 'PDB 6A9X (AnkG:GABARAP)'.

These errors have been corrected in both the PDF and HTML versions of the Article.
P. M. Jenkins

pjenkins@umich.edu

1 Department of Pharmacology, University of Michigan Medical School, 48109 Ann Arbor, MI, USA

2 Neuroscience Graduate Program, University of Michigan, 48109 Ann Arbor, MI, USA

3 Division of Life Sciences, State Key Laboratory of Molecular Neuroscience, Hong Kong University of Science and Technology, Clear Water Bay, Kowloon, Hong Kong, China

4 Department of Cell Biology, Duke University Medical Center, 27710 Durham, NC, USA
5 Departments of Biochemistry, Neurobiology, and Howard Hughes Medical Institute, Duke University Medical Center, 27710 Durham, NC, USA

6 Department of Psychiatry, University of Michigan, 48109 Ann Arbor, MI, USA

7 Department of Neurology, University of Michigan, 48109 Ann Arbor, MI, USA

8 Department of Molecular and Integrative Physiology, University of Michigan, 48109 Ann Arbor, MI, USA

9 Hefei National Laboratory for Physical Sciences at the Microscale, School of Life Sciences, University of Science and Technology of China, Hefei, China 